\title{
Blind Signal Extraction Algorithm for the License Plate Matching of Vehicle Positioning System
}

\author{
Shing Tenqchen ${ }^{12^{*}}$, Ying-Haw Shu ${ }^{1}$, Ming-Chang Sun ${ }^{1}$, Wu-Shiung Feng ** \\ ${ }^{1}$ Lab 331, Department of Electrical Engineering, National Taiwan University, Taipei, Taiwan, R.O.C. \\ *Chunghwa Telecom Telecommunication Labs., 12, Lane 551, Sec. 5 , \\ Min-Tsu Rd., Yang-Mei Zien, Tao-Yuan County, Taiwan 326, R.O.C. \\ **Department of Electronics Engineering, Chang Gung University, Taoyuan, Taiwan, R.O.C. \\ E-mail: d86921028@ntu.edu.tw and stc@cht.com.tw
}

\section{ABSTRACT -}

In this paper, we use ICALAB [6] tool with our algorithm to extract the desired image of license plate captured by sensors, which will be essential used by vehicle positioning system (VPS). The use of the blind least mean square algorithm (BLMS) can often provide a useful method to extract the sources and independent component. The BLMS learning rule has a self-normalizing property, such that the unit length of the weight vector $\mathbf{w}_{\mathbf{1}}$ is approximately maintained. Simulation results show that the proposed BLMS significantly extract the license plate number when the vehicle is driving from Lane 1 to Lane 2 with moving blur.

\section{Introduction}

Blind Source Separation (BSS) is a fundamental problem in signal processing with a large number of applications in speech processing, array signal processing, multiuser communications, etc. [1]-[3]. The BSS problem consists in recovering the original sources from the observations only, without knowing the transmitted channel and sources. Most of the existing approaches to BSS have been developed for instantaneous mixtures. In practical situations, however, this hypothesis is not true and it is more common to find convolutive mixtures. Different algorithms [2]-[3] have been proposed to separate convolutive mixtures of sources in a blind way. Although this problem has recently attracted a lot of interest because of its wide number of applications in diverse fields, BSS can be very computationally demanding if the number of source signals is large. In particular, this is the case of license plate matching applications, where it is desired to extract only some "interesting" sources. Fortunately, sequential blind signal extraction (BSE) overcomes somewhat this difficulty. The BSE problem considers the case where only a small subset of sources has to be recovered from a large number of sensor signals.

This paper is organized as follows. The signal model of the preliminary results of blind least mean square (BLMS) algorithm is presented in Section 2. Section 3 will discuss the license plate recognition process. A design example to use the ICALAB [6] tool with preprocessing and post-processing functions for our algorithm in Section 4. The simulation result of is used to prove our algorithm.

2. Signal Model: The Blind Least Mean Square (BLMS) Algorithm

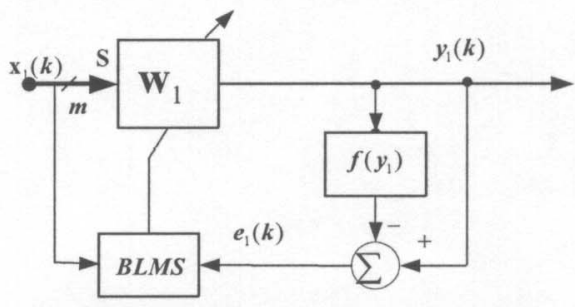

Fig.1 Implementation of BLMS algorithm.

The block diagram structure of the sequential blind extraction of sources and independent component is shown in Fig. 1. Let us consider the KuicNet algorithm developed by Kung and Douglas [2] has been derived from the normalized kurtosis but under the constraint that vector $\mathbf{w}_{\mathbf{1}}$ has unit length, i.e., $\left\|\mathbf{w}_{1}\right\|^{2}=1$

$$
\min J_{1}\left(\mathbf{w}_{1}\right)=-\frac{\beta}{4} \frac{E\left\{\boldsymbol{y}_{1}^{4}\right\}}{\left\|w_{1}\right\|^{4}},
$$

where $\beta=\operatorname{sign}\left(\boldsymbol{\kappa}_{4}\left(\boldsymbol{y}_{1}\right)\right)$ is the constant penalty factor. The standard blind least square (BLMS) algorithm with a "blind" error signal equal to $e_{1}(k)=y_{1}(k)-f\left[y_{1}(k)\right]$. It should be 
noted that

$\mathbf{w}_{1}(k+1)=\mathbf{w}_{1}(k)+\eta \beta\left(y_{1}^{3}(k) x_{1}(k)-y_{1}^{4}(k) w_{1}(k)\right)$,

where $\eta>0$ is the learning rate. It should be noted that the above KuicNet learning rule has a self-normalizing property, such that the unit length of the weight vector $\mathbf{w}_{\mathbf{1}}$ is approximately maintained. However, when the extracted source signal $\boldsymbol{y}_{1}(\boldsymbol{k})$ has a negative value of kurtosis, the above algorithm is unstable and the vector $\mathbf{w}_{1}$ must periodically renormalized to the unit length as follows

$$
\mathbf{w}_{1}^{+}(k+1)=\mathbf{w}_{1}(k)+\eta \boldsymbol{\beta}\left[y_{1}^{3} \mathbf{x}_{1}-y_{1}^{4} \mathbf{w}_{1}\right],
$$

where $\mathbf{w}_{1}(k+1)=\mathbf{w}_{1}^{+}(k+1) /\left\|\mathbf{w}_{1}^{+}(k+1)\right\|_{p}$, and where

$p$ is the 1-norm to $\infty$-norm. Alternatively, we can use the ordered rotation KuicNet learning rule proposed by [2]

$$
\begin{gathered}
\mathbf{w}_{1}(k+1)=\mathbf{w}_{1}(k)+\boldsymbol{\eta} y_{1}^{3}(k)\left[\mathbf{x}_{1}(k)-y_{1}(k) \mathbf{w}_{1}(k)\right] \\
\text { for } \boldsymbol{\beta}>0, \\
\mathbf{w}_{1}(k+1)=\mathbf{w}_{1}(k)-\boldsymbol{\eta} y_{1}^{3}(k)\left[\left\|\mathbf{w}_{1}\right\|^{4} \mathbf{x}_{1}(k)-y_{1}(k) \mathbf{w}_{1}(k)\right] \\
\text { for } \beta<0 .
\end{gathered}
$$

We can formulate this constrained optimization criterion as

$$
\min J_{1}\left(\mathbf{w}_{1}, \mathbf{b}_{1}\right)=\frac{1}{2} E\left\{\varepsilon_{1}^{2}\right\}+\frac{\beta_{1}}{4}\left(1-E\left\{y_{1}^{2}\right\}\right)^{2},
$$

where $\beta_{1}>0$ is the constant penalty factor. The standard stochastic gradient descent method leads to an on-line learning algorithm for the vector $\mathbf{w}_{1}$ and coefficients of the FIR adaptive filter $b_{\mathrm{lp}}$, respectively,

$$
\begin{aligned}
\mathbf{w}_{1}(k+1) & =\mathbf{w}_{1}(k)-\eta_{1} \frac{\partial J_{1}\left(\mathbf{w}_{1}, \mathbf{b}_{1}\right)}{\partial \mathbf{w}_{1}} \\
& =-\eta_{1}(k)\left[\left\langle\varepsilon_{1}(k) \widetilde{\mathbf{x}}_{1}(k)\right\rangle-\gamma(k) \mathbf{w}(k)\right],
\end{aligned}
$$

where $\gamma(\boldsymbol{k})=-\beta_{1}\left[1-\hat{\boldsymbol{\sigma}}_{\boldsymbol{y}_{1}}^{2}(\boldsymbol{k})\right]$ is a forgetting factor and

$$
\begin{aligned}
b_{i p}(k+1) & =b_{i p}(k)-\tilde{\eta}_{1} \frac{\partial J_{1}\left(\mathbf{w}_{1}, \mathbf{b}_{1}\right)}{\partial b_{1 p}} \\
& =\tilde{\eta}_{1}(k)\left\langle\varepsilon_{1}(k) y_{1}(k-p)\right\rangle,
\end{aligned}
$$

where $\widetilde{\mathbf{x}}_{1}(k)=\mathbf{x}_{1}(k)-\sum_{p=1}^{L} b_{1 p} \mathbf{x}_{1}(k-p)$ and $\eta_{1}$ as well as $\tilde{\eta}_{1}$ are the learning rates. The variance of output signal $\sigma_{y_{1}}^{2}=\boldsymbol{E}\left\{\boldsymbol{y}_{1}(\boldsymbol{k})^{2}\right\}$ can be estimated on-line by using

$$
\sigma=\frac{1}{2} \zeta d v\left(\frac{1}{\kappa}-\frac{1}{v}-\frac{1}{u}\right)
$$

where $\kappa$ is the focal length, $\boldsymbol{u}$ is the distance of the object point from the lens, $v$ is the distance between the lens and image detector $\boldsymbol{d}$ is the diameter of the lens aperture, and/is a camera that depends on its optics and Charge-Coupled Device (CCD) array resolution
[3]. It shall be noted, that in order to avoid the inversion of autocorrelation matrix $\mathbf{R}_{\mathbf{x} 1 \mathbf{x} 1}$ in each iteration step, we can apply as a preprocessing the standard prewhiting or standard PCA and next normalize the sensor signals to unit variance. In such cases, $\hat{\mathbf{R}}_{\mathbf{x} 1 \mathbf{x} 1}=\mathbf{I}_{n}$ and the algorithm is simplified to

$$
\mathbf{w}_{1}^{+}=\hat{\mathbf{R}}_{\mathbf{x}_{1} \hat{\mathbf{y}}_{1}} \mathbf{b}_{1}=\hat{\mathbf{R}}_{\mathbf{x}_{1} \tilde{\mathbf{y}}_{1}}, \quad \mathbf{w}_{1}=\frac{\mathbf{w}_{1}^{+}}{\left\|\mathbf{w}_{1}^{+}\right\|}
$$

where $\hat{\mathbf{R}}_{\mathbf{x}_{1} \tilde{\mathbf{y}}_{1}}=\frac{1}{N} \sum_{k=1}^{N} \mathbf{x}_{1}(k) \tilde{y}_{1}(k)$.

\section{The Recognition Process of License Plate}

The recognition process of license plate can be implemented as followed: (a) image capture, (b) image storage, (c) license plate positioning, (c) character region segmentation, (d) character segmentation, (e) character recognition, and (f) output the recognized character. The VPS-based multilane free-flow(MLFF) can be constructed by Fig. 2. The main steel frame 1 is constructed with Laser BLMS vehicle sensor detector with $6 \mathrm{~m}$ height. The detecting area was installed by adjusting sensing area in Fig. 3 . When vehicles is driven to pass the sensing area, the reflected signal is sent by DIO (digital $\mathrm{I} / \mathrm{O}$ card) to $\mathrm{CCD}$ camera to catch several vehicle images. Main steel frame 2 installed with $5 \mathrm{CCD}$ cameras is $5.43 \mathrm{~m}$ height. The capturing area is overlapped sequentially.

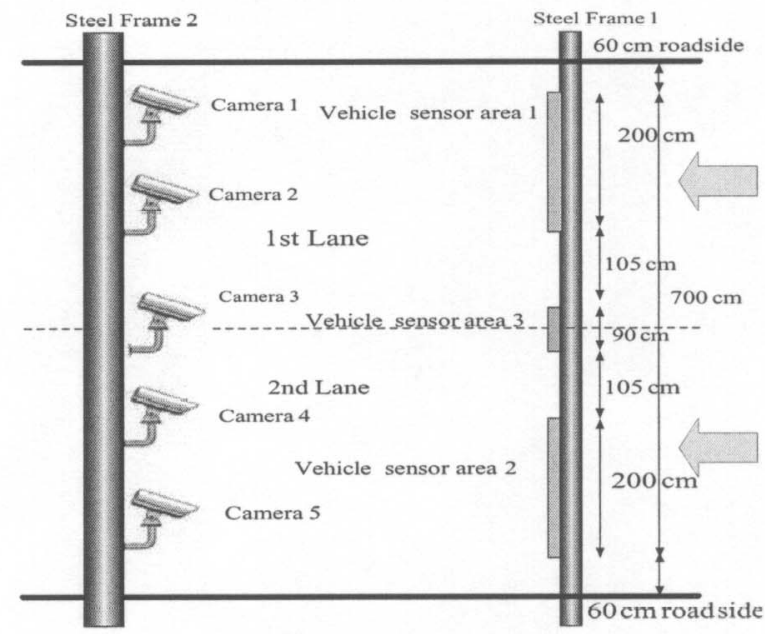

Fig 2. The installation of camera and sensors on test site. 




Fig 3. The setting area of Vehicle sensor-detector by BLMS algorithm.

\section{Design Example with License Plate Matching of}

\section{Vehicle Positioning System}

The important and unique features of this paper is to use the ICALAB [6] toolbox with preprocessing and post-processing functions. The second order statistics blind source extraction to any possible matrix factorization of the form $\mathrm{X}=\mathrm{HS}+\mathrm{N}$ or $\mathrm{Y}=W^{T} X$ where $\mathrm{H}=\mathrm{W}^{+}$is a mixing matrix. The direction of image scanning is horizontal and vertical. The processing area is whole image with random sampling. This action uses the whole image area for estimation of the demixing matrix (pixels are scanned randomly).



Fig. 4. The input of images capturing by sensors

\subsection{2}

Fig 4. The output of image for the license plate recognition.

\section{Conclusion}

In this paper, we use ICALAB [6] tool with our algorithm to extract the desired image of license plate captured by sensors. It can be noted that applying the standard linear predictor and by minimizing the least mean error $E\left\{\varepsilon^{2}\right\}$, which is measure of predictability. The observation suggests that it is not needed to minimize the cost function with respect to parameters $\left\{b_{1 \mathrm{p}}\right\}$ but it is enough to choose the arbitrary set of them for which the largest eigenvalue is unique. More generally, if all eigenvalues of the generalized covariance matrix $\mathbf{R}_{\mathbf{x}_{1}}\left(\mathbf{b}_{1}\right)$ are distinct, then we can extract all sources simultaneously by estimating principal eigenvectors of $\mathbf{R}_{\mathbf{x}_{1}}\left(\mathbf{b}_{1}\right)$.

\section{References}

[1] A. Cichocki, and S. Amari, Adaptive Blind Signal and Image Processing: Learning Algorithms and Applications, John Wiley \& Sons, LTD, 2002.

[2] S. C. Douglas and S. Y. Kung, "Kuicnet algorithms for blind deconvolution," In Proc. IEEE Workshop on Neural Networks for Signal Processing, pp. 3-12, Cambridge, UK, Aug. 1998.

[3] A. P. Pentland, "A new sense for depth of field," IEEE Trans. Pattern Anal. Machine Intell., vol.9, pp. 523-531, July 1987.

[4] S. Cruces, L. Castedo, and A. Cichocki, "Asymptotically equivariant blind source separation using cumulants," Neural computation, in print 2002.

[5] O. Shalvi and E. Weinstein, "New criteria for blind deconvolution of nonminimum phase systems (Channel)," IEEE Trans. Inform. Theory, vol. 36(2), pp. 312-320, Mar 1990.

[6] A. Cichocki, S. I. Amari, K. Siwek, "ICALAB for Signal Processing: Toolbox for ICA, BSS, BSE," http://www.bsp.brain.riken.go.jp. 